\title{
Philosophiques
}

\section{Femmes, hommes et féministes à la barre}

\section{Ariane Michelle Djossou}

Volume 21, numéro 2, automne 1994

Les femmes et la société nouvelle

URI : https://id.erudit.org/iderudit/027277ar

DOI : https://doi.org/10.7202/027277ar

Aller au sommaire du numéro

Éditeur(s)

Société de philosophie du Québec

ISSN

0316-2923 (imprimé)

1492-1391 (numérique)

Découvrir la revue

Citer ce document

Djossou, A. M. (1994). Femmes, hommes et féministes à la barre. Philosophiques, 21(2), 343-355. https://doi.org/10.7202/027277ar

\section{Résumé de l'article}

Pourquoi continue-t-on à dénigrer le féminisme malgré l'amélioration notable de la condition des femmes grâce à ses revendications ? On peut invoquer le sens péjoratif persistant du mot " féminisme ", la récupération des thèses féministes par les politicien-nes, ou encore les divergences au sein des tendances féministes. Mais ces motifs ne devraient pas empêcher la prise de conscience du fait que le féminisme ne recherche que la construction d'une société nouvelle juste pour les deux sexes. 


\title{
Femmes, hommes et féministes à la barre
}

\author{
par \\ Ariane Michelle Djossou
}

\begin{abstract}
RESUME : Pourquoi continue-t-on à dénigrer le féminisme malgré l'amélioration notable de la condition des femmes grâce à ses revendications? On peut invoquer le sens péjoratif persistant $d u$ mot «féminisme », la récupération des thèses féministes par les politicien-ne-s, ou encore les divergences au sein des tendances féministes. Mais ces motifs ne deuraient pas empêcher la prise de conscience du fait que le féminisme ne recherche que la construction d'une société nouvelle juste pour les deux sexes.
\end{abstract}

ABSTRACT : Why is feminism still denigrated in spite of notable improvements in the condition of women due to its claims? One can invoke the lasting pejorative meaning of the word « feminism », the politicians' recovery of feminist propositions, or the diverse stands of the main feminist trends. But these reasons should not prevent us to see that feminism only wants a new and just society for both women and men.

Depuis quelques décennies, la condition des femmes a connu plusieurs changements et améliorations grâce aux revendications féministes. Pourtant, pour bon nombre de personnes, tant dans les milieux populaires que chez les intellectuels des deux sexes, il reste difficile de rompre avec la fausse neutralité traditionnelle et d'admettre réellement la valeur inévitable et inéluctable du féminisme. D'une part, dans la vie quotidienne, on manifeste une résistance à voir dans le féminisme une pierre centrale pour la construction d'un monde nouveau. D'autre part, on doute de l'importance épistémologique de la recherche féministe en refusant d'accorder à la diversité des courants et tendances féministes la même valeur qu'à celle des disciplines comme la philosophie ou l'anthropologie, pour ne citer que celles-là, dont les divers courants, systèmes, écoles et représentants ne cessent d'afficher des contradictions, des affrontements, voire des exclusions. Ce manque de considération que subissent les études féministes est-il dû au fait que le féminisme ne constitue pas encore un domaine scientifique autonome, et qu'il s'insère à l'intérieur des autres branches de la connaissance ? Ou est-il dû à son fondement ? Quelles peuvent être les conséquences de ce manque de considération pour les objectifs du féminisme? Permettent-elles de construire la nouvelle société sur des 
bases solides et justes ou servent-elles seulement à la rénovation de l'ancienne? Enfin, quelle est la provenance des considérations négatives que reçoit le féminisme, si ce n'est ce qui émane des femmes, des hommes et des féministes ${ }^{\mathrm{I}}$ ? Aussi ceux-ci doivent-ils plaider coupables aux chefs d'accusation qui seront analysés. Toutefois, il est important de signaler que toute la cour est constituée par eux. Ils sont à la fois victimes, plaideurs, coupables et juges. Par conséquent, les termes du verdict se trouvent dans leurs mains.

Notre analyse se propose d'établir des jalons pour une conscientisation du féminisme contemporain comme créateur d'une pensée qui propose un monde juste dans lequel le statut d'être humain serait accordé à toutes et à tous sans discrimination. En d'autres termes, il s'agira d'une part, pour la vie quotidienne, d'éveiller toute la société humaine à la connaissance réelle du féminisme, et à la destruction de la manière courante de fuir l'adhésion officielle tout en acceptant ses avantages. D'autre part, pour les théoriciens et théoriciennes, il importe de reconnaître que la neutralité est une illusion et un mythe qui font du chercheur ou de la chercheure un être aliéné, c'est-à-dire un être qui est autre à force de vouloir sortir de lui-même pour atteindre à la pseudo-objectivité scientifique, et qui perd progressivement la base concrète de la réalité sociale, ou qui adhère inconsciemment à certains préjugés du milieu. Enfin, il suffit de comprendre que la diversité des champs d'intérêt et des méthodes en études féministes ne compromet pas les objectifs du féminisme.

\section{Ce qu'est le féminisme}

Le féminisme présenté comme une révolte, un mouvement historique, une théorie politique, ou une idéologie, est sans aucun doute inscrit dans l'histoire humaine, car qu'on soit au Nord, au Sud, à l'Est ou à l'Ouest, nul sur cette planète ne peut prétendre ignorer son existence, encore moins être neutre vis-à-vis de son évolution. Il suscite réprobations ou éloges lorsqu'il apparaît dans les journaux, les essais, les critiques, bref, dans les conversations les plus familières, voire les plus intimes de l'existence humaine. Qu'il soit désigné comme une théorie ou un mouvement, son essence est toujours la même : la cause des femmes, une cause qui se défend par des pratiques, des luttes, des discours, et dont la finalité, pour la plupart, est la transformation en profondeur de l'ordre social patriarcal et injuste. Mais si l'existence du féminisme est un fait inéluctable à notre époque, qu'évoque-t-il pour l'esprit commun et pour les féministes elles-mêmes? Car l'expérience quotidienne montre qu'il ne suffit pas d'admettre l'existence d'une chose pour que celle-ci acquière une valeur positive, encore faut-il chercher à la connaître et/ou à la

I. Pourquoi les mettre ensemble? nous dira-t-on. La réponse apparaîtra lorsque nous présenterons les contradictions qui existent au sein des féministes. 
comprendre. Peut-on dire que le féminisme est connu et compris dans les sociétés?

Assurément, certaines personnes diront un oui catégorique et pourront même prétendre que cette question, à la fin du XXe siècle, n'a plus de raison d'être; elle est dépassée : tout le monde sait ce qu'est le féminisme. Elles donneront des définitions : le féminisme, c'est le mouvement des femmes; c'est la doctrine qui lutte pour la cause des femmes, etc. Celles qui ont plus de culture recourront à des citations : on appelle féminisme

[...] toute analyse, toute action, tout geste posant comme conflictuels - et défavorables aux femmes - les rapports entre les deux sexes et visant à en comprendre la nature ou à en modifier les termes ${ }^{2}$; lou :l

par le terme féminisme, nous désignons l'ensemble plus restreint de discours et de pratiques qui donne priorité à la lutte des femmes et qui pose comme finalité l'abolition, du moins la transformation en profondeur, de l'ordre patriarcal ${ }^{3}$.

Les plus éclairées ajouteront qu'en matière de définition du féminisme, il vaut mieux se référer aux auteures, aux ouvrages qui présentent les conceptions de plusieurs tendances, plutôt qu'aux dictionnaires. Dans tous les cas, ce qui ressort est que l'ordre socio-sexuel est discriminant et injuste envers les femmes, et, qu'être féministe, c'est avoir un préjugé favorable à l'égard des femmes. Or, en prenant pour acquis que l'objectif fondamental du féminisme est la création d'un monde où la femme et l'homme seront considérés également, peut-on dire que la société globale, les femmes, les hommes et même les féministes, réagissent favorablement aux divers modèles proposés ? Est-il possible d'y répondre positivement en analysant ces réflexions quelque peu contradictoires?

\section{Se libérer oui... Être féministe non.}

Madame Claudette constate que la libération des femmes est une nécessité pour lutter contre la discrimination, cependant elle se refuse à être prise pour une féministe. Elle affirme: « Je suis une femme, je reconnais qu'il $y$ a beaucoup de discrimination sexuelle dans notre société et que les femmes doivent se libérer, mais je ne suis pas une féministe ».

Madame Danielle s'indigne de l'inégalité des salaires qui prévaut dans son lieu de travail, mais elle ne veut pas que cette indignation soit confondue avec une réaction féministe. Elle dit : « Je ne suis pas d'accord avec la disparité salariale que l'industrie textile impose aux hommes et aux femmes; mais je ne suis pas féministe... ».

2. M. Albistur et D. Armogathe, L'histoire du féminisme français, Paris, Des Femmes, 1978 , p. 9.

3. F. Descarries-Bélanger et S. Roy, Le mouvement des femmes et ses courants de pensée : essai de typologie, Les Documents de l'ICREF, 1988, n' 19, p. 2. 
De son côté, Madame Ginette éduque et encourage sa fille et son garçon pareillement; mais selon elle, être féministe n'est pas nécessaire : « Je suis femme et mère, j'ai un bon emploi; je n'accorde pas de valeur ou d'appréciation inégale au développement psychique de mon fils et de ma fille. Pour moi, tous deux sont pareils; l'un n'est pas plus important que l'autre, et je pense qu'on peut bien vivre dans la société actuelle sans être féministe... ».

D'une enquête faite dans les écoles secondaires du Québec, il ressort que les jeunes filles rencontrent des difficultés dans le monde adolescent lorsqu'elles s'affirment féministes. Selon Lise Julien ${ }^{4}$, les réflexions négatives et dévalorisantes dérangent les jeunes filles plus que le harcèlement sexuel : «Montrer que tu es féministe, c'est la tare par excellence », lui confie une adolescente. Une autre lui dit : «Tu peux à la rigueur favoriser des valeurs égalitaires, mais si tu t'affiches féministe, t'es finie ».

Plusieurs personnes ont compris et excusé la réaction de Nathalie Provost en face de Marc Lépine qui, criant « je hais les féministes », joint le geste à la parole et tue 14 femmes de l'École polytechnique le 6 Décembre ig8g à Montréal : «On n'est pas des féministes, on est juste des filles qui veulent étudier », avait-elle répondu. D'autres y ont vu la phrase magique qui peut sauver - ne pas être féministe - , et prétendent qu'à la place de Nathalie, toute féministe convaincue aurait certainement renoncé à son manteau féministe pour avoir la vie sauve. La projection à l'Université Laval du film $A$ u-delà du 6 décembre, de Catherine Fol, n'a pas contredit cette dernière réflexion:

L'héroïne, Nathalie, décide de continuer de vivre et de se battre pour occuper la place qui lui revient. Elle ne se considère pas comme une féministe mais comme un être humain qui a toute l'existence devant elle pour trouver le bonheur et la vérité $e^{5}$.

commente l'une des rares femmes qui se sont exprimées sur ce qu'elles ont ressenti en visionnant le film.

Pour les partis politiques, c'est connu, le féminisme est devenu la recette la plus rapide de l'opportunisme; supporter les revendications féministes et promettre beaucoup de changement ainsi qu'une diminution des charges des femmes, est un moyen de s'attirer une bonne partie du vote féminin. Il ne faut surtout pas chercher à voir si toutes les promesses se réalisent ou non. De plus,

4. Lise Julien, « Si tu t'affiches féministe, t'es finie », dans La Gazette des Femmes, 1986. vol. 8, n I, p. 33 .

5. Renée Larochelle, « Au-delà du 6 décembre....La vie » dans Au fil des Evénements. Université Laval, vol. 27, n̊14, 5 Décembre 1991, p.7. C'est nous qui soulignons. Evidemment, il est courant d'entendre des femmes qui s'adonnent aujourd'hui à des activités non traditionnelles dire qu'elles ne sont pas féministes. D'un certain point de vue, elles ont raison, puisqu'elles n'ont pas lutté pour obtenir cela. Que leurs grand-mères et mères l'aient fait ou non, ne dérange en rien ces propos. L'histoire atteste que lorsqu'un acquis entre dans les mœurs, il devient une habitude, et est très vite considéré comme « normal »... 
les femmes en politique sont plus favorables que les hommes aux demandes des mouvements féministes concernant l'autonomie et les droits des femmes sur leur corps et leur santé. Mais malgré les nombreux recoupements des demandes des organisations féministes, et malgré les actions des politiciennes, ces dernières ne suivent pas toujours les cheminements qu'attendent les féministes. Selon Manon Tremblay, bien que les politiciennes «entretiennent une image péjorative des féministes, souvent associées au modèle caricatural de la "radicale" », elles n'en posent pas moins des actions féministes. De plus, «les élues doivent s'adapter à l'environnement politique $»^{6}$.

La liste des refus d'être féministe est longue. Pourtant, si on demandait à celles qui supportent mal la disparité salariale et à celles qui accordent la même valeur au développement de leurs enfants de préciser en quoi leurs besoins sont originaux par rapports à ceux que revendiquent les féministes, elles se lanceraient dans des « oui, mais moi... ». Autrement dit, si on posait la question du comment et du pourquoi aux partisanes et partisans de ces réflexions, ils se retrouveraient nécessairement devant le féminisme. Qu'y a-t-il de si inquiétant et de méprisant dans le fait d'être féministe et de (se) l'avouer? A quel jeu joue la société lorsqu'elle catégorise ainsi les féministes? Les normes, les comportements et les lois reformulés sous le poids des pressions féministes sont-ils inutiles au point qu'il faille éviter d'affirmer ses victoires?

L'enjeu de ces interrogations est d'ordre implicite et explicite, car le refus de toute critique n'empêche pas les tenantes et tenants des remarques mentionnées plus haut, de bénéficier des fruits des revendications féministes. Que d'actions pouvons-nous rappeler pour montrer les réalisations du féminisme dans le monde ${ }^{7}$. L'éducation et l'instruction sont désormais ouvertes aux

6. Manon Tremblay, Les fermmes en politique représentent-elles les femmes? De quelques conduites des femmes et des hommes en politique au Québec à l'endroit des demandes exprimées par les mouvements féministes. Thèse de Doctorat, Université Laval, I99I, p. 294. Cette façon d'excuser les politiciennes revient à dire que le fait d'être dans un groupe d'hommes oblige une femme à adopter les expressions masculines dans son langage, et qu'en politique les femmes devraient parler la langue des hommes, comme le conclut Lise Payette dans Le pouvoir, connais pas! Dans cet ordre d'idées, les théoriciennes et les chercheures en sciences humaines ne feraientelles pas mieux de suivre les traces traditionnelles de la « connaissance de l'homme », en admettant sans critique que l'homme dont il est question n'est pas le mâle mais l'humain, donc homme et femme à la fois ?

Nous pensons que de nos jours, dans un groupe quelconque ou dans un parti politique, il ne devrait y avoir aucune excuse pour cette manière d'adhérer au langage courant, même quand on est conscient de la présence inhérente de l'injustice. Car le fait d'utiliser le langage traditionnel ne permet pas un changement ou une transformation réels.

7. Il faut, bien entendu, restreindre cet argument aux pays les moins dominés par les religions. 
femmes, de l'école primaire à l'université; les barrières professionnelles sont de plus en plus flexibles pour les femmes; il existe de moins en moins d'emplois uniquement réservés aux hommes et interdits aux femmes. Les femmes mariées ont le droit d'exercer un emploi lucratif sans autorisation maritale; la possibilité de limiter le nombre d'enfants, d'intervenir sur son propre corps et de contrôler sa propre fécondité, est désormais en voie d'être acquise. L'esprit critique des femmes refuse d'être cloîtré dans les choses dites privées. La solidarité féminine s'accroît pour briser la société patriarcale qui, pendant des siècles, a sécrété la discrimination à l'égard des femmes, et qui est en phase de disparition ou, du moins, en transition vers la nouvelle société.

Aussi, pour celles et ceux qui le constatent, le féminisme est devenu, en certaines occasions, le moyen de sauvegarde de la vie, d'un poste, d'une certaine féminitè, d'une certaine différence, d'une naturalité de femelle opposée à la naturalité mâle ou à l'imitation du mâle. Le féminisme tend à être une levure : son absence ou sa présence peuvent apporter de bons résultats ${ }^{8}$; on s'en sert selon les circonstances et les lieux. On est féministe quand il y a abolition d'une barrière discriminatoire; on se défend de l'être lorsque les revendications se confrontent à beaucoup d'obstacles et suscitent trop d'opposition. Au moment où la féminisation des titres permet aux femmes de se sentir à l'aise dans le poste qu'elles occupent, sans donner l'impression qu'elles investissent le domaine du mâle, il est bien dommage pour les chercheures linguistes et grammairiennes féministes de voir qu'il existe des femmes qui refusent l'usage des termes féminisés. Dans certaines municipalités, les femmes refusent d'être nommées mairesses et préfèrent être appelées maires, bien que la première appellation soit recommandée par l'Office de la langue française. Dans cette optique, si certaines femmes ont autant de difficultés à croire en la positivité du féminisme, qu'attendre des hommes?

Certains séparent les femmes des féministes, ou refusent de nommer leur épouse une féministe: « Écoute donc, ce n'est pas une femme celle-là, c'est une féministe... », crie Pierre à son ami qui se plaint du partage des tâches domestiques que sa compagne lui demande.

Dans une salle de détente en résidence étudiante, les titres d'un prochain journal télévisé annoncent que « le plus grand mouvement des femmes au pays affirme que les ententes constitutionnelles ne tiennent pas compte de $52 \%$ de la population : les femmes », et plusieurs voix d'hommes questionnent: «Que veulent-elles encore, ces féministes? 》.

8. Cela dépend du lieu d'où on l'utilise et aussi des buts qu'on poursuit. La jeune femme qui refuse d'être féministe en face de son copain pour ne pas le choquer ou le perdre, redevient vite féministe devant ses parents si ces derniers pensent qu'il y a des disciplines pour garçons et qu'il y en a d'autres pour les filles, qui seraient naturellement inaptes à celles des hommes. 
Pour cet ouvrier en bâtiment, il faut croire ce que dit son épouse : « Mon épouse occupe un très bon poste dans l'industrie textile; c'est une passionnée de la question des revendications féminines, mais elle n'est pas une féministe, elle-même le dit... ».

Pour Monsieur Jacques, c'est autre chose; il affirme son opposition à l'insertion des femmes dans des domaines traditionnellement réservés aux hommes : « Je veux bien que les femmes aient un travail rémunérateur, mais je m'oppose à ce qu'une femme usurpe une place d'homme. Et puis que feront les hommes si les femmes prennent tous les emplois sans distinction? Franchement... ».

Et cet étranger qui rencontre des difficultés pour courtiser les femmes à Québec, de s'exclamer : « Vous les féministes, vous enlevez à la femme tout ce qu'elle possède de naturel, et ici, tout est harcèlement sexuel ».

Il apparait que ces réflexions populaires, aussi futiles ou ridicules qu'elles paraissent, ne doivent pas être prises à la légère; car c'est dans le quotidien que se forme la structure d'une transformation des mentalités. De plus, les propos les plus courants sont ceux que véhicule la grande masse de la population, et l'histoire montre combien ils peuvent se traduire dans des actes jugés objectifs, c'est-à-dire faits par les plus « éclairés » de la société. Certains parmi les dirigeants et dirigeantes, les journalistes et tous ceux et celles qui ont un certain pouvoir de communication, continuent d'exercer des pressions négatives sur les changements de mentalité au sujet du féminisme.

Dans la presse écrite et et la presse orale, on trouve des usages du terme « féminisme » soit pour montrer combien les comportements d'une féministe relèvent du non-ordinaire, soit pour dire que les féministes n'apparaissent que pour troubler le bon déroulement des choses. Autrement dit, le féminisme sert à donner du piquant à une affaire, variable suivant les occasions. Que n'a-t-on pas lu au sujet du projet de loi 146 de la ministre Gagnon-Tremblay ? Certaines organisations féministes comme le Réseau d'Action et d'Information pour les Femmes (RAIF) proclamaient lajustesse du projet, alors que d'autres personnes le critiquaient comme discriminatoire pour une majorité de femmes. Pour Benoît Lauzière, qui s'est vigoureusement attaqué au projet, même les amendements apportés après coup « ne corrigent pas l'erreur de départ ». Le projet de loi I46, affirme-t-il, est « la stratégie des professionnelles d'une certaine idéologie féministe... »9. Par contre, pour Lysiane Gagnon, c'est « un projet qui lèse toutes les femmes au travail ». Ce projet, ajoute-t-elle, n'est pas féministe et il prouve combien la ministre Gagnon-Tremblay a une vue rétrograde du mariage. Le mariage, en 1989, n'est plus celui d'avant; car si chaque personne a le droit de faire un contrat avec un fournisseur, avec un employeur, pourquoi

9. Le Devoir du 22 Juin 1989. Souligné par nous. 
n'aurait-on pas le droit de faire un contrat avec son mari ${ }^{\mathrm{IO}}$ ? Quel acte doit-on considérer comme féministe ? Est-ce ce qui touche les femmes les plus démunies de la société ou ce qui touche celles qui savent déjà comment s'exprimer et faire valoir leurs droits?

Notre analyse ne vise pas la résolution de ce problème. En le présentant, nous voulons attirer l'attention sur le fait que le féminisme sert selon les circonstances. Il n'est aucun doute que l'acte posé par la politicienne vise une certaine catégorie de femmes et que, de ce fait, il est non traditionnel en politique. Néanmoins, si cet acte favorise certaines personnes, il demeure discriminant pour d'autres; et, bien entendu, il entraîne des contradictions et des oppositions entre femmes, entre femmes et hommes, et entre féministes.

\section{Le féminisme se vend, il donne du piquant aux recettes....}

Le magazine La Vie en Rose, avant sa disparition, a affirmé qu'il était le seul magazine féministe d'actualité au Québec. Une publicité à laquelle s'oppose, à juste titre, le RAIF. Car dire qu'on est « le seul magazine féministe » dans un milieu où il en existe beaucoup d'autres, c'est non seulement s'arroger la clientèle, mais surtout, c'est occulter les autres revues et bulletins féministes qui s'auto-financent. Les numéros 95/96 (mars-avril Ig86) de la Revue du RAIF, consacrés à cette occultation, se présentent comme une véritable querelle qui ne permet pas un regard social positif sur le féminisme; d'autant plus que lors de la table ronde organisée plus tard par La Vie en Rose - probablement dans l'intention de corriger son erreur -, et à laquelle est conviée toute la presse féministe du Québec, Communiqu'elles et la Revue du RAIF refusent de participer pour cause de mésentente idéologique ${ }^{\mathrm{II}}$. Mais la vente du féminisme n'est pas l'apanage de revues féministes, car même les journaux qui semblent les plus inoffensifs se servent du féminisme pour « apprêter leurs plats ». Voici ce qui est écrit dans un magazine de cinéma de Québec : « Une jeune veuve s'engage dans l'armée et se démarque au cours de grandes manœuvres. Scénario dispersé. Mise en scène assez alerte. Clichés troupiers servis à la sauce féministe..... $\gg^{12}$.

Io. La Presse du i5 Juillet 1989. Une féministe convaincue et peut-être radicale, pensera$t$-on, mais prudence, il n'en est rien. Elle-même se classe dans la tendance modérée. (p.222 infra). Qu'on se souvienne de la crainte de cette auteure au sujet de l'éducation des petites filles élevées par des mères féministes au Québec dans Viure avec les hommes... Montréal, Québec-Amérique, 1983. Qu'adviendra-t-il de ces enfants, se demande-t-elle, quand leurs mères « auront refusé de leur apprendre l'art subtil de séduire et de se conformer tout en faisant à sa tête ? Ne risquentelles pas de se retrouver un jour totalement sans défense, comme des chats dégriffés lâchés dans la jungle » (p. 145)?

II. Pelletier, F., « La presse féministe au Québec. A chacune son créneau », dans La Vie en Rose, n’36, Mai 1986, p.I8.

12. Résumé du film La Bidasse de H. Zieff, dans Télé-Soleil, semaine du 6 au 12 juin 1992, p. 14. C'est nous qui soulignons. 
Il existe beaucoup d'autres propos de ce genre dans la presse. Nous n'allons pas, pour ce travail, faire des statistiques sur l'appétit qu'éveillent les plats servis à la sauce féministe; qu'il nous suffise de dire que l'accueil du féminisme ou ce qu'il représente en ce moment n'est pas dénué d'ambiguité. Quand une femme réagit contre une injustice, elle perd son statut de femme et devient féministe. Lorsqu'elle affiche son côté affectif et sentimental, ses collaborateurs disent d'elle qu'elle est une femme... Mais si elle évite de montrer la "sensibilité traditionnelle des femmes », les pleurs, on préfère la nommer « femme de fer ». On crée des expressions pour montrer combien ces actions sont contraires à l'ordinaire. Puisque toutes les femmes n'ont pas les mêmes comportements, celles qui se démarquent méritent d'être qualifiées autrement. Mais comment ? La qualification est presque toujours négative. Dans tous les cas, le qualificatif retire ce qui est considéré comme traditionnellement « positif » pour la femme.

Dans une telle situation, bien que l'instruction des filles et des garçons soit générale et obligatoire, il n'y a pas de doute que dans la jeune génération on accueille cet état de choses comme normal. Les gouvernants et les législateurs ayant fini par accorder un certain nombre de revendications féministes dans leurs lois et actes, plus personne, comme nous le précisions plus haut, ne se souvient du fait que tout cela a été et est fait grâce au féminisme, l'optimisme actuel étant que le féminisme militant a déjà cédé la place à un féminisme tranquille qui va tellement de soi qu'il n'a plus à se définir ou à s'affirmer.

De plus, il faut affirmer que l'ambiguité du terme « féminisme » reste perceptible lorsqu'on regarde de près ce qu'on en fait. Qui, de nos jours, n'a jamais été confronté à une situation où tout doit se faire et se dire avec un grand souci d'éviter de choquer l'auditoire par l'usage du mot « féminisme »? Certaines organisations non gouvernementales s'en tirent par le simple recours au mot « femme ». D'autres précisent leur humanisme, qui, selon eux, concerne autant la femme mariée et la célibataire, que la mère et l'enfant. En ce sens, présenter une requête en y incluant le terme « féminisme », cela signifie, en certaines occasions, la signature de son échec ou de sa réussite. Et ainsi, le féminisme, c'est bien l'utilité non proclamée. C'est le remède dont l'identification nominale, au lieu d'attirer, fait plutôt fuir. En d'autres termes, le féminisme, c'est la potion indispensable au changement de l'ordre patriarcal, mais dont le nom trouble et inquiète. Et pourtant, chaque femme a ses objectifs.

Selon les enquêtes de C. Lord et M. Vezina ${ }^{13}$, le féminisme se morcelle; les femmes luttent individuellement sans chercher à intégrer les groupes. Les femmes entrepreneures s'imposent de plus en plus sur le marché au Québec. Même si toutes les femmes ne militent pas dans des organisations féministes, presque toutes ont des objectifs à atteindre et se prononcent de leur lieu

13. La Gazette des Femmes, 1987, vol. 8, n 6, p. II. 
d'action ou en fonction de leurs besoins : sortir de la dépendance, avoir la sécurité économique, réaménager le marché du travail, développer des services, aider l'entrée sur le marché du travail, se donner du temps à soi, favoriser la formation des filles, rejoindre les femmes non regroupées, orienter les découvertes scientifiques, exercer son droit au travail, prendre toute sa place, sortir de la pauvreté, alimenter la réflexion, trouver des stratégies, exiger l'accès à l'égalité, etc. Ainsi, dans les conversations et dans la vie quotidienne, le féminisme a une connotation péjorative, et il vaut mieux vivre, de plus en plus, en suivant des objectifs qu'on croit personnels alors qu'ils rejoignent bien ceux du féminisme organisé. Mais qu'en est-il du monde théorique?

\section{Le féminisme professionnel}

De ce côté, tout semble positif : « La recherche féministe se porte bien », affirme Lucie Pinault ${ }^{14}$. Cette recherche non seulement se fait, mais encore est fructueuse, si l'on se reporte aux colloques organisés par les groupes de recherches féministes comme le GREMF de l'Université Laval. Certes, ce constat est juste et valable pour plusieurs autres groupes du pays dans la mesure où,

[...] en se plaçant du point de vue des femmes, point de vue occulté jusquelà, la recherche féministe déplace l'angle d'observation, élargit les problématiques et contribue à combler les vides et les lacunes du savoir concernant les femmes et les rapports sociaux de sexe. IEt d'lun strict point de vue de recherche, elle augmente la qualité des connaissances et permet d'approfondir la compréhension de la réalité sociale.

De plus, les chercheuses féministes, reconnaissant que la neutralité en science est un mythe, ne cachent pas leur parti-pris pour les femmes; en conséquence, elles se préoccupent de la pertinence de leurs projets et des retombées qu'ils auront sur les femmes ${ }^{15}$.

Pourtant, c'est une autre impression que donnent les études féministes. Car ce qu'on constate dans la diversité des méthodes et des approches des chercheures est une sorte de « champ de bataille ».

En effet, des courants et des tendances naissent et tracent leur itinéraire comme à l'intérieur d'une discipline traditionnelle. La seule différence, qui n'est pas la moindre, est que leurs débats ne semblent pas être pris pour un enrichissement, mais plutôt pour un manque de cohésion et d'entente. On se critique, on s'oppose et on s'exclut, non pas seulement à cause des approches et des conceptions différentes, mais aussi à cause de la différence sexuelle. On rencontre l'approche fẻministe hétérosexuelle, l'approche féministe lesbienne, l'approche féministe matérialiste, raciale, culturelle, religieuse, écologique, etc. qui se greffent sur les grands courants libéraux, socialistes, marxistes ou radicaux. Le féminisme libéral situe l'oppression des femmes

14. La Gazette des Femmes, I987, vol. 8, n'6, p. 9.

15. Dagenais, Huguette, « Pour les femmes, un autre développement », dans Recherches Féministes, vol. I, n 2, 1988, p. 4. 
dans la discrimination dont elles sont victimes; l'homme en tant que tel n'est pas l'ennemi. Ce qu'il faut, c'est la liberté et l'égalité qui mettent fin à toute forme de discrimination. Pour le féminisme radical, l'oppression des femmes est la source de toutes les autres oppressions; c'est le pouvoir des hommes qui est la première oppression. Il faut donc mettre fin à la domination des hommes. Par contre, les féministes socialistes et surtout marxistes se prétendent plus originales et plus actives dans leurs luttes contre le patriarcat que les féministes libérales, bien qu'elles subordonnent l'oppression sexuelle à l'oppression classuelle : le mouvement des femmes doit s'intégrer à l'ensemble de la lutte révolutionnaire et fonctionner dans le cadre de la lutte des classes. Ce faisant, le courant socialiste, par exemple, rejette tous les autres modèles et valorise sa seule vision d'un monde juste. On observe aussi l'approche féministe féminine qui diffère de l'approche féministe de la féminitude. Alors que pour cette dernière, l'exaltation de la nature féminine est à l'œuvre, pour l'autre, il est essentiel de refuser aux hommes, les mâles de l'espèce humaine, la possibilité de penser et d'écrire quelque chose d'objectif sur les femmes. Être un homme devient pour ainsi dire incompatible avec l'essence du féminisme. Dans ce cas, on affirme : «c'est un écrit d'homme, il ne peut être féministe », ou « être homme et féministe, avouons-le, c'est contradictoire, non... »; ou encore, «nous ne publions pas les textes d'hommes même si les sujets traités portent sur les femmes; tout texte écrit sur les femmes n'est pas féministe... » ${ }^{\mathrm{I} 6}$.

L'un des derniers sujets qui suscitent beaucoup d'intérêt à l'heure actuelle, est la préoccupation pour les Nouvelles Technologies de Reproduction Humaine (NTRH). Si, pour certaines féministes, les NTRH favorisent l'exploitation des femmes pauvres par les riches, pour C. Delphy ${ }^{17}$, les NTRH constituent un problème réel, mais il n'est pas particulièrement féministe. Selon la féministe matérialiste, il s'agit là d'un problème général entre la population et le corps médical. Et la situation des prostituées est bien plus désespérante que celle des femmes qui vendent leur corps pour être des mèresporteuses. Des milliers de femmes sont des prostituées sans le vouloir; elles sont séquestrées, enfermées et vendues quelquefois par leurs parents; elles vivent dans des conditions d'esclavage et vendent leur corps en tout temps et

I6. Certaines personnes à l'Université Laval ont été confrontées à ces refus de la part de revues féministes. Assurément, ce n'est pas parce qu'un ouvrage porte dans son titre le mot «femme » qu'il est féministe, l'histoire de la philosophie nous l'apprend. Mais le refus de l'objectivité aux hommes à propos des femmes devraitil, à l'époque actuelle, avoir pour raison leur appartenance au groupe sexuel masculin ? N'y-a-t-il pas là un risque de sexisme au féminin?

I7. Delphy, Christine, « La revendication maternelle », dans Descarries-Bélanger, F. et al. Questionnements et pratiques de recherches féministes, Montréal, UQAM. Centre de Recherche Féministe, 1990, p. I2. 
pas même à leur profit. L'exploitation des mères porteuses ne dure que neuf mois; le fruit de leur travail leur revient et pire, leur condition est voulue.

Par une pareille analyse, Delphy minimise la portée du discours féministe qui s'oppose aux NTRH. Evoquer comme argument que ce problème général est celui de la population et du corps médical est une manière d'écarter les féministes de la population. Affirmer aussi que les opposantes aux NTRH ont une « peur non fondée et même absurde » et que leur inquiétude fait « penser que les femmes ne servent qu'à la procréation ${ }^{18}{ }^{18}$, c'est permettre des controverses sur des préoccupations sociales sérieuses. Ainsi, la diversité des tendances du féminisme, qui se présente comme un véritable « champ de bataille », ne participe pas toujours à une conception favorable de la finalité du féminisme, contrairement à ce qu'on devrait attendre. Dans la mesure où le fondement de l'oppression est basé pour certaines tendances sur le sexe, et que, pour d'autres, il l'est sur la race et/ou la classe, il ne nous reste qu'à privilégier les propositions et les formes de sociétés qui découlent des courants et des tendances. On devrait ainsi voir quel type de société s'accorderait le mieux aux besoins humains. Serait-ce une sociēté réformée, c'est-à-dire qui a subi une transformation partielle, ou une société androgyne anarchiste, ou non anarchiste, ou une société gynocratique, ou encore une société gynocentrique, etc. ? Ainsi, la possibilité de construire la nouvelle société, cette société naissante, qui ne semble pas s'affranchir de l'empreinte des dernières décennies, pourra appartenir à toutes et à tous, et chaque personne saura éviter les préjugés.

\section{Conclusion}

Somme toute, il ressort des évocations des propos, des réflexions et des actes des femmes, des hommes et des féministes, que la considération donnée à une pensée, une théorie ou une action dans une société, reste dépendante de la capacité de ses membres. Une société qui se veut juste ne saurait émettre des avis défavorables sur les apports de la théorie féministe tout en acceptant les avantages qui s'y rattachent. En ce sens, le tribunal que nous avons ouvert au début de notre analyse invite, après délibération, à une conscientisation du féminisme contemporain, non pas seulement comme une forme de révolte et de désaccord, mais aussi comme une acceptation consciente de la capacité de création d'une pensée qui, sans prétendre à son tour discriminer, violenter ou soumettre un sexe à l'autre, propose une justice sociale fondée sur le statut d'être humain de toutes et de tous. Ainsi, la diversité des courants féministes qui, pour la masse sociale, femmes, hommes et féministes, est un lieu de contradiction, d'exclusion, voire de mésentente entre leurs membres, serait plutôt le lieu privilégié de discours et de débats théoriques mais s'appuyant sur du concret. Par cela, la multitude des tendances féministes aurait droit au

18. Delphy, ibid, p. г3. 
même respect intellectuel et scientifique que la multitude des systèmes qu'on rencontre par exemple en philosophie, puisque d'une époque à l'autre, et souvent dans la même époque, l'histoire de cette discipline si respectée présente des points de vue divers et opposés sur un même problème. A aucun moment ces diverses conceptions philosophiques n'ont reçu l'accueil « méprisant » et « diffamatoire » que reçoit le féminisme. Certes, il ne s'agit pas pour nous de nier les éléments de remise en cause violente ou « agressive » de certaines tendances féministes axées sur l'action ${ }^{19}$. Cependant, à y regarder de plus près, aucune idéologie, aucune théorie sociale ou politique ne manque de détracteurs, car les extrémistes existent partout. Or cette face hétérogène ne sert pas pour autant à catégoriser et à sous-estimer les théories socio-politiques. Elle ne doit donc pas servir à dévaloriser le féminisme. Le respect de la pensée de toutes et de tous est d'une importance capitale dans notre construction d'une société nouvelle.

Aussi les féministes et toute la société doivent-elles en appeler à l'esprit constructeur qui fait du féminisme la seule pensée humaine qui rejette d'emblée la fausse neutralité traditionnelle utilisée pour masquer les prises de position injustes. Il est donc urgent que les femmes et les hommes, en ce début des années quatre-vingt-dix, prennent conscience de la reconnaissance du féminisme comme valeur inévitable et inéluctable pour la construction d'un monde juste.

19. Valérie Solanas est un exemple de l'extrémisme qu'on rencontre dans tout mouvement de libération ou de révolte. Dans son livre SCUM Manifesto, New York: The Olympia Press, ig68, elle affirme que haïr les hommes et même les exterminer est un acte bon et droit, un acte hautement bénéfique aux femmes en même temps qu'un acte de pitié (p. 67). 